\section{Putting a Sphere on an Atomic Force Microscope Cantilever Tip}

Stefan Zauscher, University of Wisconsin Madison

Atomic Force Microscopes (AFM) can measure the force between a surface and the tip of a cantilever as a junction of separation with great precision. For example, van der Waals type forces and electrostatic repulsive forces can easily be measured in aqueous solutions using an AFM. The complex, pyramidal shape of the typical AFM cantilever is, however, not well suited for quantitative measurements. It is thus desirable to attach particles of known geometry (usually spheres) to the tip of a cantilever.

This technique, pioneered by Ducker et al. in $1992^{1}$, has led to a number of investigations where particle modified cantilevers were used (for example, refs. 2-4), and has led to the publication of a support note addressing the procedure of how to attach particles to cantilevers ${ }^{5}$. The adhesive of choice seems to be an epoxy resin solid (Epon 1004) manufactured by Shell. Epon 1004 is used as a hot melt adhesive and requires heating the cantilevers on a hotstage. This can lead to warping of (especially metal coated) cantilevers.

In my work, I also need to attach spherical particles (cellulose beads) onto standard silicone nitride AFM cantilevers, but I need to avoid excessive heat. I found that an optical adhesive which cures in seconds when exposed to ultraviolet light, and thus does not need heat treatment for crosslinking, works very well. Norland's optical adhesive \#81 (NOA \#81) is designed for bonding applications where low strain, optical clarity and low outgassing are required ${ }^{6}$. NOA \#8I has a viscosity of $300 \mathrm{cp}$ at $25^{\circ} \mathrm{C}$, which allows dispensing the adhesive from a micro-pipette with a tip diameter of less than $0.2 \mu \mathrm{m}$. The adhesive has a modulus of $1.4 \mathrm{GPa}$ (about $200,000 \mathrm{psi}$ ), a Shore $D$ hardness of 90 , and cures extremely fast (within 60 seconds) when exposed to longwave (320 to $380 \mathrm{~nm}$ ) UV light ${ }^{6}$. A small hand held or desk top UV light source can be used.

Cantilevers deflect (droop and twist) under the weight of the object attached to the free end. A nice way to observe the distortion in a lever (Figure) is to use a light microscope equipped with Nomarski interference. This differential contrast technique is used in light microscopy to emphasize surface structure. Using this observation method, I find that cantilevers are sometimes already distorted when new and I usually discard these levers. The Nomarski technique may also offer a non-destructive way to calibrate the lever for stiffness by placing particles of known mass on the end of the lever and observing the color change with deflection. Color video images of the deflected lever could easily be analyzed with image analysis software and, once a color deflection calibration has been performed, the lever stiffness can be inferred. (I haven't investigated the feasibility of this yet).

It is imperative when attaching particles to make sure that the particle is absolutely centered on the lever to minimize twisting of the lever under contact pressure or in a strong force field. The twisting motion of a cantilever is usually coupled to the deflection motion of the cantilever, and thus twisting can give rise to an undesirable deflection. The strength of this coupling and any unusual twist while the cantilever is brought into contact with the surface can be observed with an AFM where both horizontal and vertical deflection signal of the lever can be accessed simultaneously. For example, with Digital Instruments' MultiMode AFM and Software version 4.22, turn on Channel 2 in the force calibration mode and set it to friction ${ }^{7}$. The lateral force signal should not change dramatically upon engaging the tip.

\section{References:}

1. Ducker, W. A; Senden, T. J.; Pashley, R. M. Measurement of Forces in Liquids Using A Force Microscope. Langmulr, 1992, 8, 1831-1836.

2. Butt, $H_{\text {.; }}$ Jaschke, M.; Ducker, W. A. Measuring surface forces in aqueous electrolyte solution with the atomic torce microscope. Bioelectrochemistry and Bioenergetics, 1995, 38, 191-204.
3. Biggs, S. Steric and Bridging Forces between Surfaces Bearing Absorbed Polymer: An Atomic Force Microscopy Study. Langmuir, 1995, 11, 156-162. 4. Braithwaite, G. J. C. and Luckham, P. F. Study of Attractive Interactions Between Poly(ethylene-oxide) Coated Surfaces Using AFM. In: Proceedings of the NATO Advanced Study Institute on Micro/Nanotribology and its Applications (Sesimbra, Portugal, June 16-18, 1996), Bhushan, B. ed. Kluwer Academic Publishers, Dordrecht, NL. 121-127.

5. DIgital Instruments. Attaching Particles to AFM Cantilevers. Support Note No. 226, Rev. A. Digital Instruments. 112 Robin Hill Rd.. Santa Barbara CA, 93117. 1996.

6. Norland Products, 695 Joyce Kilmer Ave., New Brunswick, NJ 08902, phone: 908-545-7828; website: http://www.norlandprod.com

7. Digital Instruments, 112 Robin Hill Rd., Santa Barbara CA, 93117, phone:

800-873-9750: website: http://www.di.com

\section{Tips On Finding A Good Etchant For Metals:}

\section{Bernard Kestel, Argonne National Laboratory}

Start with a handbook of chemistry and physics. In the tables of physical constants will be listed the metals solubilities in various acids. Pick an appropriate acid, and as a first try use $10 \%$ acid in methanol (a good electrical conductor). Ethanol is less conductive and more viscous than methanol. Always check chemical safety before starting. Never mix nitric acid with ethanol, for example; it explodes above a $5 \%$ concentration. Chill the solvent to at least $0^{\circ} \mathrm{C}$ before adding acid.

Refinements may start with adjusting the etching bath temperature to $0^{\circ}$ $\mathrm{C}$ or $-45^{\circ} \mathrm{C}$. Also, 2 to $5 \%$ butyl cellusolve may improve the polishing film, assisting the preservation of precipitates and boundaries, while smoothing the matrix.

\section{An example:}

Etching copper from cobalt may be possible with a 3-5\% mixture of nitric acid in methanol or water. Try using the mixture at room temperature; if the action is too slow, increase the acid concentration.

This approach may work because copper is dissolved by nitric acid (and electropolished by mixtures containing nitric acid). The CRC Handbook lists cobalt as soluble in acids, but with no specifics. Electropolishing mixtures for cobalt contain percholric acid, not nitric. So, nitric acid may have little effect on cobalt, and dissolve copper.

Another approach to etching is to use a non-acid electrolyte. I have developed one called BK-2. This solution does not cause hydride formation in materials initially free of hydride. (It doesn't work on copper, however.)

This solution is less hazardous than the usual perchoric acid solution, and generally does not attack dislocations around large precipitates, unlike acid electrolytes. This attack usually causes the loss of the precipitate from the sample, as the surrounding matrix is etched away.

BK-2 is made as follows:

$$
\begin{aligned}
& 5.3 \mathrm{~g} \mathrm{LiCl} \\
& 11.16 \mathrm{~g} \mathrm{Mg}\left(\mathrm{C} 1 \mathrm{O}_{4}\right) 2 \\
& 100 \mathrm{~mL} \text { butyl cellusolve } \\
& 500 \mathrm{~mL} \text { methanol }
\end{aligned}
$$

Try using in a single-jet electo-polishing unit at $-50^{\circ}$ to $-60^{\circ} \mathrm{C}$ and 80 to $200 \mathrm{~V}, 15-20 \mathrm{~mA}$, with slow to medium electrolyte flow through the nozzle, depending on the sample.

A difficult sample of Al with 6 wt- $\% \mathrm{Ge}$, furnace aged to grow large precipitates was thinned by the above solution, to which had been added 200 $\mathrm{mL}$ glacial acetic acid. Thinning was done at $-25^{\circ} \mathrm{C}, 100 \mathrm{~V}$, and $15 \mathrm{~mA}$ in a single-jet electro-polisher.

Kestel, B.J., 1986, Non-acid electrolyte thins many materials for TEM without causing hydride formation. Ultramicroscopy 19:205-212. 